\title{
Adaptive Nonlinear Compensation for CDMA Communication Systems
}

\author{
Francisco Javier González-Serrano, Member, IEEE, and Juan José Murillo-Fuentes, Student Member, IEEE
}

\begin{abstract}
This paper deals with nonlinear compensation in code-division multiple-access communications systems. We analyze the performance of the generalized cerebellar model articulation controller neural network in two problems: predistortion in nonlinear transmitters and adaptive interference cancellation in multiuser and multipath scenarios. Our examples demonstrate the performance of the compensators in typical mobile channels.
\end{abstract}

Index Terms-Adaptive decision feedback equalization, cerebellar model arithmetic computer (CMAC), code division multiple access (CDMA), nonlinear distortion, wireless communications.

\section{INTRODUCTION}

$\mathbf{T}$ HE PERFORMANCE of the generalized cerebellar model articulation controller (GCMAC) neural network is analyzed in two nonlinear problems: predistortion and optimal detection.

In mobile environments, power-efficient amplification is a mandatory restriction. Unfortunately, power-efficient amplification means nonlinear distortion. Nonlinear distortion has three major effects on the transmitted signal.

1) The received symbols are no longer on the original regular lattice but on a warped one.

2) The combination of the nonlinear amplifier and the TX and RX pulse shaping filters does not produce a response free of intersymbol interference.

3) The nonlinear amplification causes the frequency spreading of the transmitted pulse.

These effects can be effectively reduced by means of adaptive predistorters [1], which try to produce the inverse function of the power amplifier. Under static working conditions, predistortion can be implemented off-line. However, in some cases, adaptive approaches are mandatory. Examples are the code-division multiple access (CDMA) transmitters, which use adaptive transmitter power control (TPC) to solve the near-far problem and to increase the system capacity; and cellular systems, where a base-station (BS) failure is compensated by increasing the transmitted power in the neighboring BS.

At the receiver end, we address the adaptive cancellation of the effects produced by multiple users and multipath fading on a CDMA signal. There is an intensive research effort to solve the problem of developing advanced receivers that improve the

Manuscript received June 7, 1999; revised November 11, 1999. This work was supported in part by the CICYT, PETRI, under 95-0320-QP.

The authors are with the Departamento Tecnologías de las Comunicaciones, Universidad Carlos III, Madrid 28911 Spain (e-mail: fran@tsc.uc3m.es; murillo@tsc.uc3m.es).

Publisher Item Identifier S 0018-9545(01)01143-4. performance and capacity of CDMA systems (see [2 and references therein]). In this paper, we investigate a GCMAC-based decision feedback scheme for the mitigation of multiple access interference (MAI) in CDMA wireless networks. The considered receiver approximates a Bayesian receiver and computes the GCMAC parameters with the aid of a training sequence prior to transmission. Again, an adaptive receiver solution is considered since it has to cope with time-varying channels.

This paper is organized as follows. The problem statement and notation are included in Section II. The predistorter and the receiver structures used in this paper are described in Section III. In Section IV, we include the basic essentials of the GCMAC network. Section $\mathrm{V}$ is devoted to the performance evaluation of the proposed schemes. A summary and some conclusions are given in Section VI.

\section{Problem Statement}

In CDMA communication, each user is distinguished by a unique spreading code to modulate binary antipodal data. The signal sent over the shared spectrum channel is the sum of the individual users' signals. Definitions of several key terms used in this text about CDMA systems can be found in [2].

\section{A. Communication System Model}

Consider a CDMA transmitter using complex-valued data symbols. The transmitted baseband signal in a single symbol interval $T_{s}$ can be represented as

$$
x(t)=\sum_{l=1}^{L} \sum_{n=1}^{N} \chi_{n} b_{n} \boldsymbol{c}_{n}(l) g\left(t-l T_{c}\right), \quad 0 \leq t \leq T_{s}
$$

where

$L \quad$ length of the spreading code;

$N \quad$ number of active users;

$\chi_{n} \quad$ scaling factor specified by the power-control loop;

$b_{n} \quad$ complex symbol transmitted by user $n$;

$g(t)$ transmitter pulse shaping filter;

$T_{c} \quad$ chip period. ${ }^{1}$

The spreading code of user $n$ is denoted by $\boldsymbol{c}_{n}$. Note that individual chips in $c_{n}$ are denoted by the elements in $\left[c_{n}(1), \ldots, c_{n}(L)\right]^{T}$ [superscript $(T)$ denotes transpose] with each $c_{n}(\cdot) \in \pm 1$.

In the context of our work, two impairments are incorporated in the system model. The first one is due to the nonlinear behavior of the power amplifier (PA). The nonlinear distortion of a PA is described at baseband by a memoryless nonlinearity that,

\footnotetext{
${ }^{1}$ In the UMTS standard [3], $b_{n} \in\{ \pm 1, \pm j\}, g(t)$ is a squared-root raised cosine with rolloff factor $\beta=0.22$, and $1 / T_{c}=3.84$ Mchips/s.
} 
in our analysis, obeys the Saleh model [4]. The second one is small-scale fading [5] including time spreading and time variance.

We use a discrete model with $M_{P}$ paths to simulate the time dispersion

$$
h_{n}(t)=\sum_{m=1}^{M_{P}} \alpha_{n, m} \delta\left(t-\tau_{n, m}\right)
$$

where the path gain $\alpha_{n, m}$ is a random parameter with variance specified by a intensity profile and $\tau_{n, m}$ is the time delay. In particular, we analyze the case when the received multipath components extend beyond the chip's time duration (frequency-selective fading $\left.\Rightarrow \max \left(\tau_{n, m}\right)>T_{c}\right)$. We simulate the channel time variance by changing the coefficients after a given number of bits.

\section{COMPEnSATION Methods}

Two compensation methods are considered: predistortion, to reduce the effects of nonlinear amplification, and equalization (including adaptive interference suppression), to detect the desired user data after multipath fading.

\section{A. Data Predistortion}

A data predistorter transforms the extended constellation formed by the linear combination of complex symbols $b_{n}$

$$
\Omega_{a}=\left\{a, \text { with } a=\sum_{n=1}^{N} \chi_{n} b_{n}\right\}
$$

into a new constellation $\Omega_{\tilde{a}}=\left\{\tilde{a}\right.$, with $\left.\tilde{a}=H_{\mathrm{dp}}(a)\right\}$, where $H_{\mathrm{dp}}(\cdot)$ is the predistorter function. Since the linear transmitter and receiver pulse-shaping filters produce time dependencies between the received samples, we provide the predistorter with memory. This way, the predistorted sample $\tilde{a}[k]$ at time $k$ is given by

$$
\begin{aligned}
\tilde{a}[k] & =H_{\mathrm{dp}}\left(a\left[k-N_{b}\right], \ldots, a[k], \ldots, a\left[k+M_{f}\right]\right) \\
& =H_{\mathrm{dp}}\left(\boldsymbol{a}_{P}[k]\right)
\end{aligned}
$$

where $P=N_{b}+M_{f}+1$ is the order of the predistorter. The predistorter function $H_{\mathrm{dp}}(\cdot)$ has to be designed in such a way that after linear filtering and nonlinear amplification in the transmitter, the constellation of the received samples (in absence of channel distortion) would match (or approximate) the constellation of symbols $\Omega_{a}$. To cope with changes in the operation point of the power amplifier, adaptive predistortion is mandatory. Adaptive predistortion requires the implementation of a (simplified) local receiver at the transmitter. After chip-matched shaping filtering (the remaining square-root raised cosine filter) and chip-rate sampling, the signal at the output of the local receiver yields

$$
\hat{r}[k]=F(\ldots, \tilde{a}[k-1], \tilde{a}[k], \tilde{a}[k+1], \ldots)
$$

where $F(\cdot)$ is the nonlinear mapping, which describes the behavior of the transmitter. Obviously, the objective of the predistorter is to find the symbols $\tilde{a}[k]$ that, for instance, minimize the square error $|a[k]-\hat{r}[k]|^{2}$.
Chip-rate predistorters have a limited influence on the transmitted signal spectrum: only frequencies below $1 / 2 T_{c}$ can be controlled. To achieve control over the whole transmitted spectrum, it is necessary to treat the data predistorter and the shaping filter jointly. One solution is to predistort the transmitted symbols at higher rate than the chip rate. The objective is that the (locally) received signal approximates the ideal, not only at the maximum eye-opening instants $n T_{c}$ but also at the intermediate instants $k T_{c} / \lambda=k T^{\prime}$, where $\lambda$ is an integer that satisfies $\lambda \geq 2$. This system, which provides distortion-free communication at all $k T_{c} / \lambda$ instants, is called the fractionally spaced data predistorter (FSDP) [6]-[8]. The block diagram of the FSDP is depicted in Fig. 1.

\section{B. Receivers for CDMA}

For simplicity, we consider a coherent synchronous CDMA transmission $\left(\tau_{n, 0}=0\right)$. After chip-matched shaping filtering and chip-rate sampling, we have the discrete-time observation

$$
\boldsymbol{r}[k]=\sum_{n=1}^{N} A_{n} b_{n}[k] c_{n}+z[k]
$$

where

$\boldsymbol{r}[k]=\quad$ vector of chip-rate samples taken during

$[r(1), \ldots, r(L)]_{k}^{T}$ the $k$ th symbol interval;

$A_{n} \quad$ received amplitude of user $n$;

$b_{n}[k] \quad$ symbol transmitted at time $k$;

$z[k] \quad$ uncorrelated noise.

As usual, we assume that the signal and noise are independent and zero mean.

Generally, a bank of matched filters (MFs) to the spreading codes of the $N$ users provides sufficient statistics to perform the optimum multiuser detection of transmitted bits. The resulting vector of observables is given by

$$
\boldsymbol{y}[k]=\boldsymbol{R} \boldsymbol{A} \boldsymbol{b}[k]+\boldsymbol{n}
$$

where $\boldsymbol{R}$ is the cross-correlation of spreading codes $\boldsymbol{c}_{n}, \boldsymbol{A}=$ $\operatorname{diag}\left\{A_{1}, \ldots, A_{N}\right\}$, and $\boldsymbol{b}[k]=\left[b_{1}[k], \ldots, b_{N}[k]\right]^{T}$ and $\boldsymbol{n}$ is a (correlated) noise vector.

1) Linear Receivers: The conventional linear detector performs the decision directly on the elements of $\boldsymbol{y}[k]$. This receiver exhibits a highly anomalous behavior: the near-far problem. As a result, its performance drops dramatically when the number of active users increases. More effective solutions are based on the decorrelation of spreading codes, and on the minimization of the mean square error (MSE) for each user [2].

2) Bayesian Receiver: In this paper, we focus on the minimization of the error probability. Suppose we observe a vector $\boldsymbol{r}$ in a certain interval $0 \leq t \leq T_{s}$. The optimal decision boundary between adjacent symbols $b^{(i)}$ and $b^{(i+1)}$ is defined by those vectors $\boldsymbol{y}$ that satisfy

$$
P\left(b^{(i)} \mid \boldsymbol{r}\right)=P\left(b^{(i+1)} \mid \boldsymbol{r}\right)
$$

Since the desired symbol is embedded in additive Gaussian noise, MAI [see (8)], and nonlinear distortion, possibly reduced by transmitter compensation, the optimal decision boundaries 


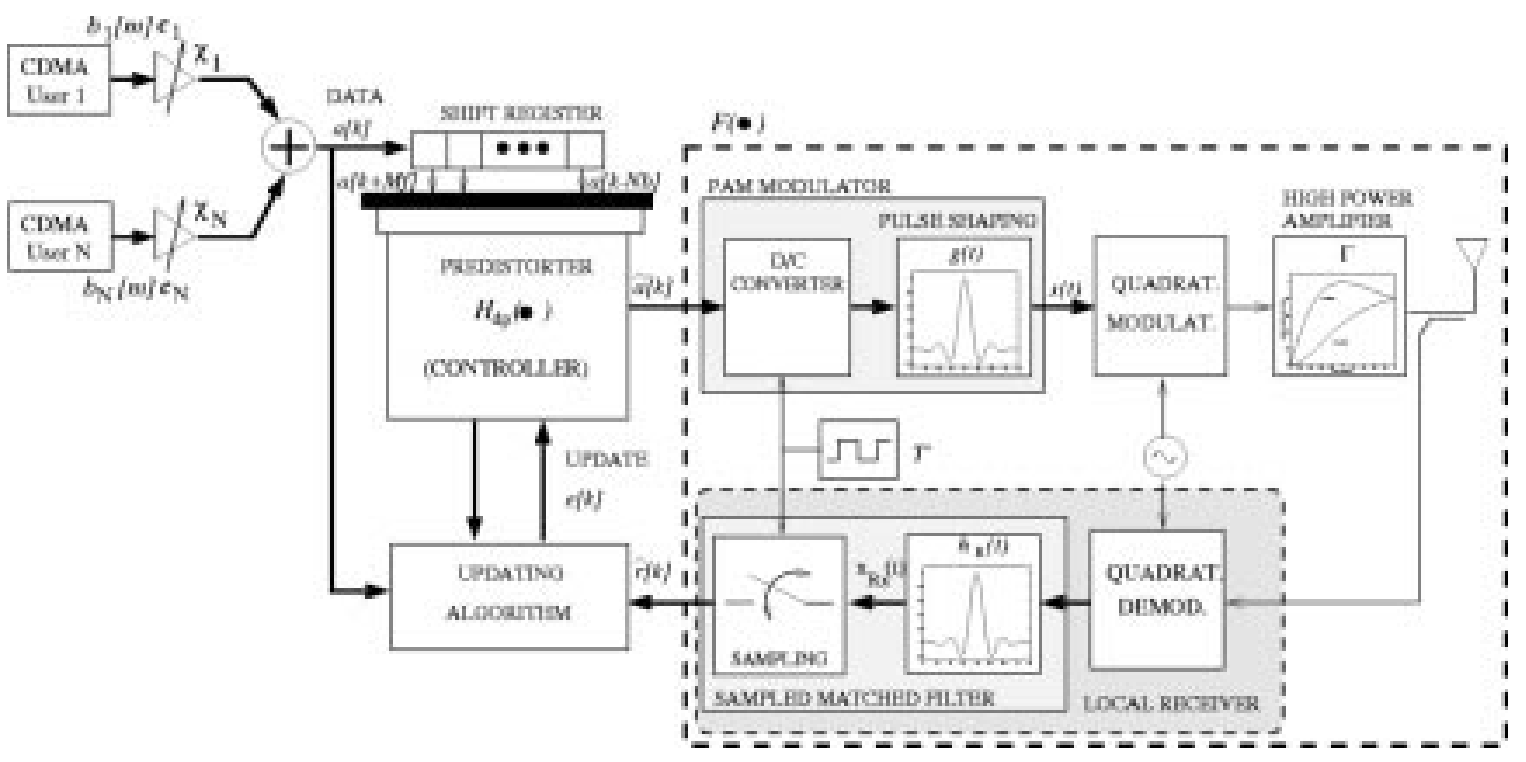

Fig. 1. Model of the CDMA transmission system with fractionally spaced data predistortion.

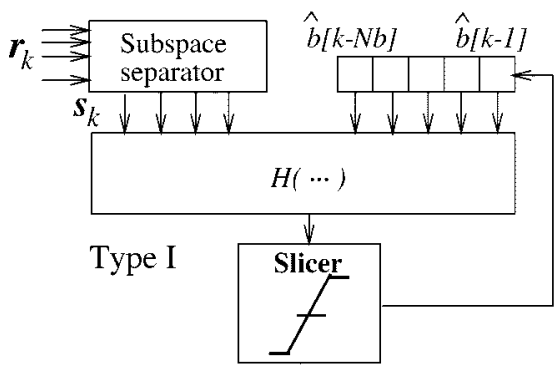

(a)

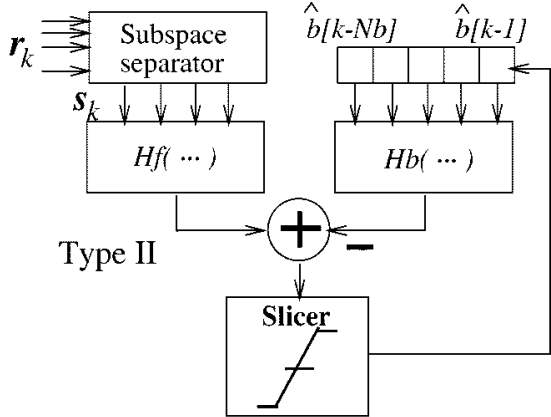

(b)

Fig. 2. Decision feedback equalizer architectures: (a) type I and (b) type II.

are highly nonlinear. For this reason, we should use nonlinear receivers.

We propose two receiver schemes inspired in the decision feedback equalizer (DFE) structure: the general DFE [DFE type I; see Fig. 2(a)] and a simplified version [DFE type II; see Fig. 2(b)].

The proposed receivers include a subspace separator that reduces both the dimensionality of input vectors and the noise correlation produced by the matched filter. It is modeled by a separating matrix of dimension $L \times N$ (obviously, $N<L$ ).

The separating matrix can be identified by an eigenvalue decomposition of the sample correlation matrix and subsequent partition into signal and noise terms. However, the performance of this method is not satisfactory for relatively high MAI [9]. Instead, we use the adaptive subspace separator proposed in [10]. Our choice is motivated by two reasons: it is more robust against MAI and is much less complex than the eigenvalue decomposition.

Both Type I and II DFEs transform the feedforward and the feedback symbols using the nonlinear functions $H(\cdot)$, $H_{f}(\cdot)$, and $H_{b}(\cdot)$, which, in our approach, are modeled by the GCMAC neural network. The main reason for its use is its potential adaptability in a time-variant communication scenario.

\section{THE GCMAC NETWORK}

Predistortion and adaptive interference suppression, or generally equalization, can be considered as a nonlinear function approximation problem. Among the special features of the compensation functions, we point out their multidimensional input space (the compensators must include memory to cope with time dependencies) and their smoothness (the ideal compensation functions take similar values at points that are close, in a Euclidean sense, in the input space).

A simple and robust approach for approximating the ideal compensation functions is the GCMAC network [11], [12]. The GCMAC performs the approximation by adjusting the amplitude of a set of local basis functions (LBFs) in the form

$$
o=H\left(\boldsymbol{w}, \boldsymbol{x}_{P}\right)=\sum_{j=1}^{M} w_{j} \Phi_{j}\left(\boldsymbol{x}_{P}\right)=\boldsymbol{w}^{T} \boldsymbol{\Phi}\left(\boldsymbol{x}_{P}\right)
$$

where $\left\{\Phi_{1}(\cdot), \ldots, \Phi_{M}(\cdot)\right\}$ is the set of LBFs and $\boldsymbol{w}$ is a weighting vector. In equalization, the input vector $\boldsymbol{x}_{P} \equiv \boldsymbol{s}_{P}$, where

$$
\boldsymbol{s}_{\Gamma}[k]=\left[s_{k}(1), \ldots, s_{k}(N), \hat{b}[k-1], \ldots, \hat{b}\left[k-N_{b}\right]\right]
$$




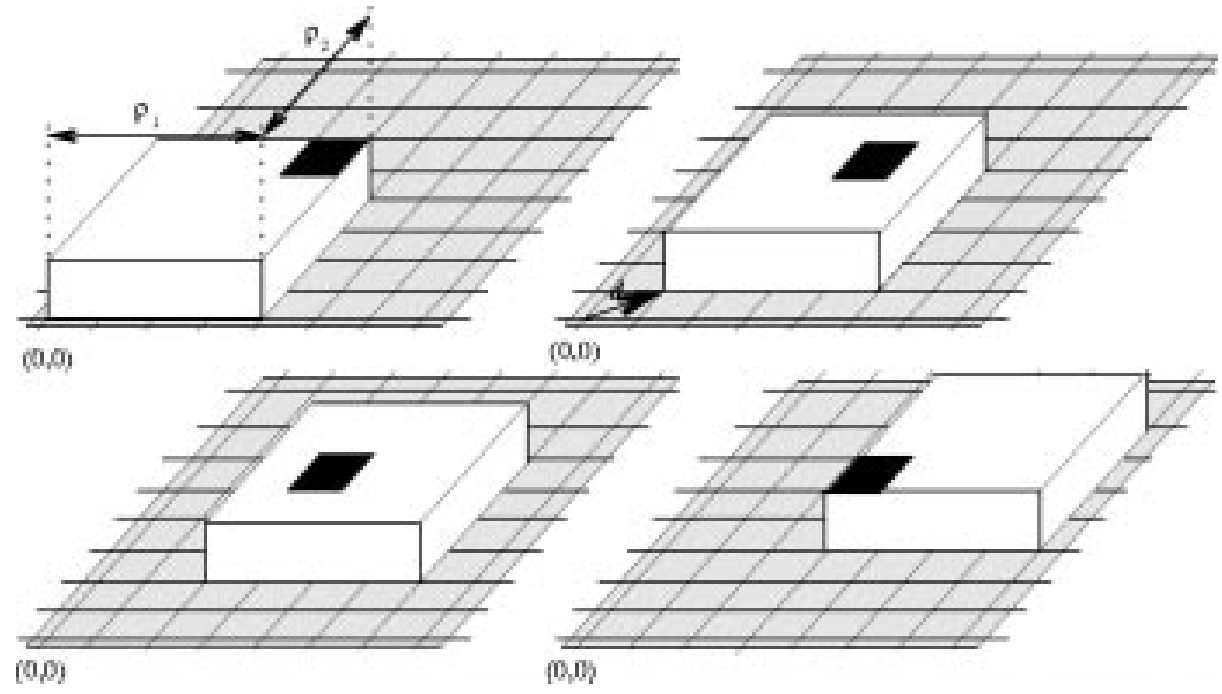

Fig. 3. Distribution of LBFs in a bidimensional space $(\rho=[4,4])$.

and in predistortion $\boldsymbol{x}_{P} \equiv \boldsymbol{a}_{P}$, where

$$
\boldsymbol{a}_{P}[k]=\left[a\left[k+M_{f}\right], \ldots, a\left[k-N_{b}\right]\right]
$$

The supports of GCMAC's LBFs are (hyper)rectangles placed at the knots of a quantization lattice ${ }^{2}$ that divides the input space into $Q^{P}$ unit cells. The size (expressed in cells) of the rectangular domains is determined by an integer-valued vector $\boldsymbol{\rho}=\left(\rho_{1}, \ldots, \rho_{P}\right)^{T}$. Therefore, unlike the radial basis functions networks, the size and location of these LBFs are predefined at the initialization step. The distribution of the LBFs across the quantized input space satisfies the following rules

1) Different quantized input samples must address different LBFs.

2) The number of LBFs that cover each input cell is a constant $\rho_{\max }=\max \left(\rho_{1}, \ldots, \rho_{P}\right)$ (when $\rho_{i}=1, \forall i$, the GCMAC becomes a lookup table).

3) The subsets of LBFs corresponding to two adjacent quantized values must only differ in one LBF (or, in other words, two adjacent cells must share $\rho_{\max }-1 \mathrm{LBFs}$ ).

The distribution of LBFs in a bidimensional space is shown in Fig. $3(\boldsymbol{\rho}=[4,4])$.

As a consequence of the last point, the integer-valued vector $\boldsymbol{\rho}$ determines not only the size of the LBFs but also the overlap between them. The result is that the vector $\boldsymbol{\rho}$ determines the generalization abilities of the network; for this reason it is called the generalization vector.

If the LBFs are constant, the GCMAC can be viewed as an indirect addressing algorithm (see Fig. 4). The indexes of the LBFs corresponding to each input cell are addresses that can be stored in a read-only memory. The amplitude of each LBF is stored on a random-access memory (RAM). This way, the GCMAC's output is obtained by adding $\rho_{\max }$ values stored in the RAM.

${ }^{2}$ The quantization is not necessary when the input symbols are discrete in amplitude, as occurs in data predistortion, and in the feedback part of a DFE.
In the update mode, the contents of the addressed memory cells can be iteratively improved according to the first-order learning law

$$
\boldsymbol{w}[k+1]=\boldsymbol{w}[k]-\frac{\mu}{2} \nabla_{\boldsymbol{w}} J(\epsilon[k])
$$

where $\mu$ is the learning parameter and $J(\epsilon)$ some cost function.

If the cost function is the square error, the learning rule is given by

$$
\boldsymbol{w}[k+1]=\boldsymbol{w}[k]+\mu e[k] \boldsymbol{\Phi}\left(\boldsymbol{x}_{\Gamma}[k]\right)
$$

where $e[k]$ is the error in the approximation. If the LBFs are constant, the vector $\boldsymbol{\Phi}\left(\boldsymbol{x}_{P}\right)$ points the $\rho_{\max }$ RAM addresses that are updated when the input is $\boldsymbol{x}_{P}$. Notice that the GCMAC algorithm requires only $2 \times \rho_{\max }$ complex sums per processed sample. The reduced computational cost, which is independent of the order $P$, makes the hardware development easier, allowing a general-purpose digital signal-processing (DSP)-based implementation [13].

\section{A. Improved GCMAC-Based Equalizers}

Even though the computational cost of GCMAC does not depend on the order, its convergence is mainly influenced by the input space dimension: the larger the order $P$, the slower the convergence (curse of dimensionality). This feature may be problematic in the equalization of mobile channels, where time dispersion can be relatively long.

To circumvent this difficulty, we have used a committee machine architecture [14] to process the feedback data symbols. Our approach is based on the linear combination of the output of two "experts" with low complexity: an $N_{b}^{\prime}$ th-order linear finite impulse response (FIR) filter and a $P$ th-order $\left(P=N+N_{b}\right)$ GCMAC network. Notice that the Volterra filter architecture has a strong resemblance to the proposed committee machine. In the following, we refer to this committee machine simply as GCMAC.

In order to achieve an accurate approximation of the optimum Bayesian boundaries, we have included an additional 


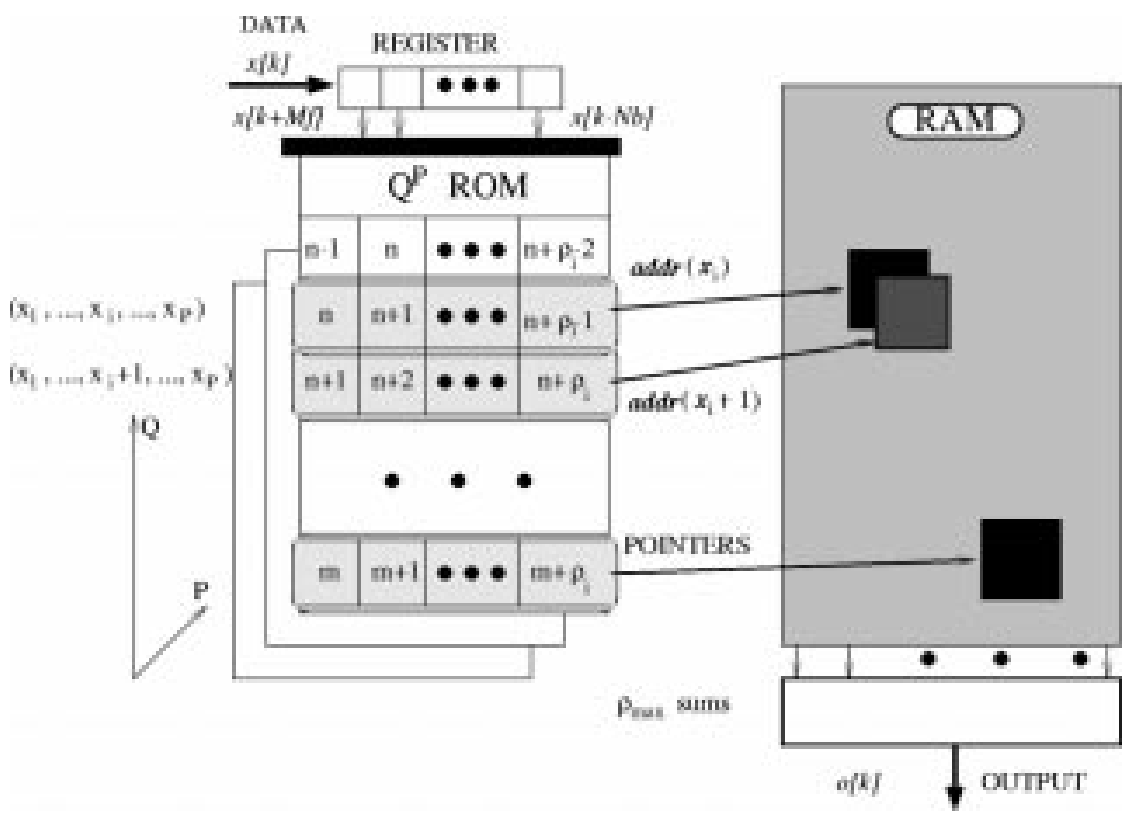

Fig. 4. Hardware implementation of the GCMAC network.

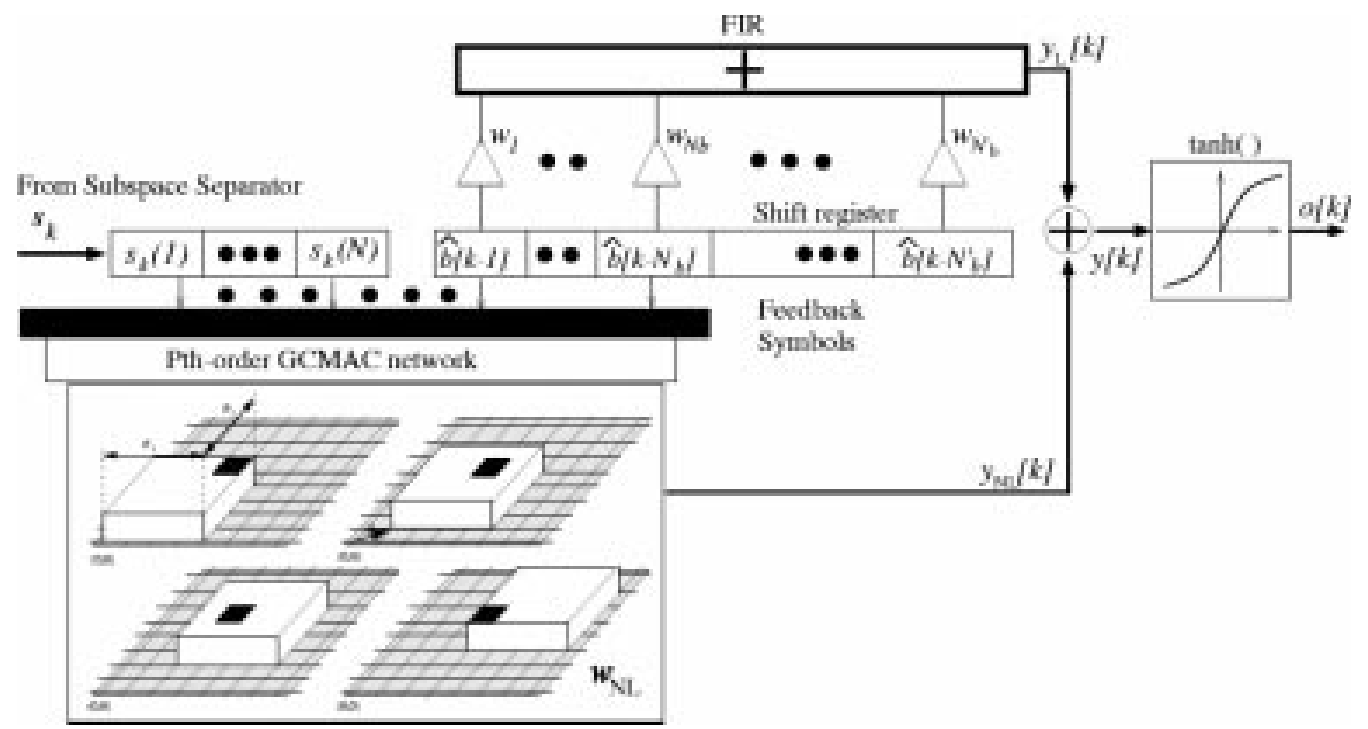

Fig. 5. Type I DFE with improved convergence. It consists of a linear FIR in parallel with a GCMAC network.

sigmoidal-like nonlinear transformation (see Fig. 5). This way, the output of the GCMAC network can be written as

$$
\begin{aligned}
o[k] & =\tanh \left(\sum_{m=1}^{M} w_{N L, m} \Phi_{m}\left(\boldsymbol{s}_{P}[k]\right)+\sum_{m=1}^{N_{b}^{\prime}} w_{L, m} \hat{b}[k-m]\right) \\
& =\tanh \left(\boldsymbol{w}_{N L}^{T} \boldsymbol{\Phi}_{N L}\left(\boldsymbol{s}_{P}\right)+\boldsymbol{w}_{L}^{T} \boldsymbol{\Phi}_{L}\left(\hat{\boldsymbol{b}}_{N_{b}^{\prime}}\right)\right) .
\end{aligned}
$$

The individual weights $\left(w_{N L}\right.$ and $\left.w_{L}\right)$ have to be updated taking into account the sigmoidal nonlinearity. If the cost function is the square error, we have

$$
\boldsymbol{w}_{\bullet}[k+1]=\boldsymbol{w}_{\bullet}[k]+\mu e[k]\left(1-o^{2}[k]\right) \boldsymbol{\Phi}_{\bullet}
$$

where $\boldsymbol{w}_{\bullet}$ denotes either $\boldsymbol{w}_{L}$ or $\boldsymbol{w}_{N L}$. The use of the hyperbolic tangent at the GCMAC output allows the use of cost functions based on the relative entropy (Kullback-Leibler). These cost functions provide a higher reduction in the bit error probability. In this case, the learning rule can be written as

$$
\boldsymbol{w}_{\bullet}[k+1]=\boldsymbol{w}_{\bullet}[k]+\mu e[k] \Phi_{\bullet} .
$$

\section{PERformance AnAlysis}

We have simulated an $N$-user CDMA system. The spreading codes are Gold codes of length $L=31$ with normalized crosscorrelations $R_{i, j}=(1 / 31) \boldsymbol{c}_{i} \boldsymbol{c}_{j}^{T} \leq 7 / 31$. The baseband modulation is quaternary phase-shift keyed (QPSK) with complex data symbols and root-raised cosine pulse shaping filter $(\alpha=$ $0.22)$. The chip rate is $1 / T_{c}=3.84 \mathrm{Mchips} / \mathrm{s}$ and the carrier frequency is $2 \mathrm{GHz}$. 


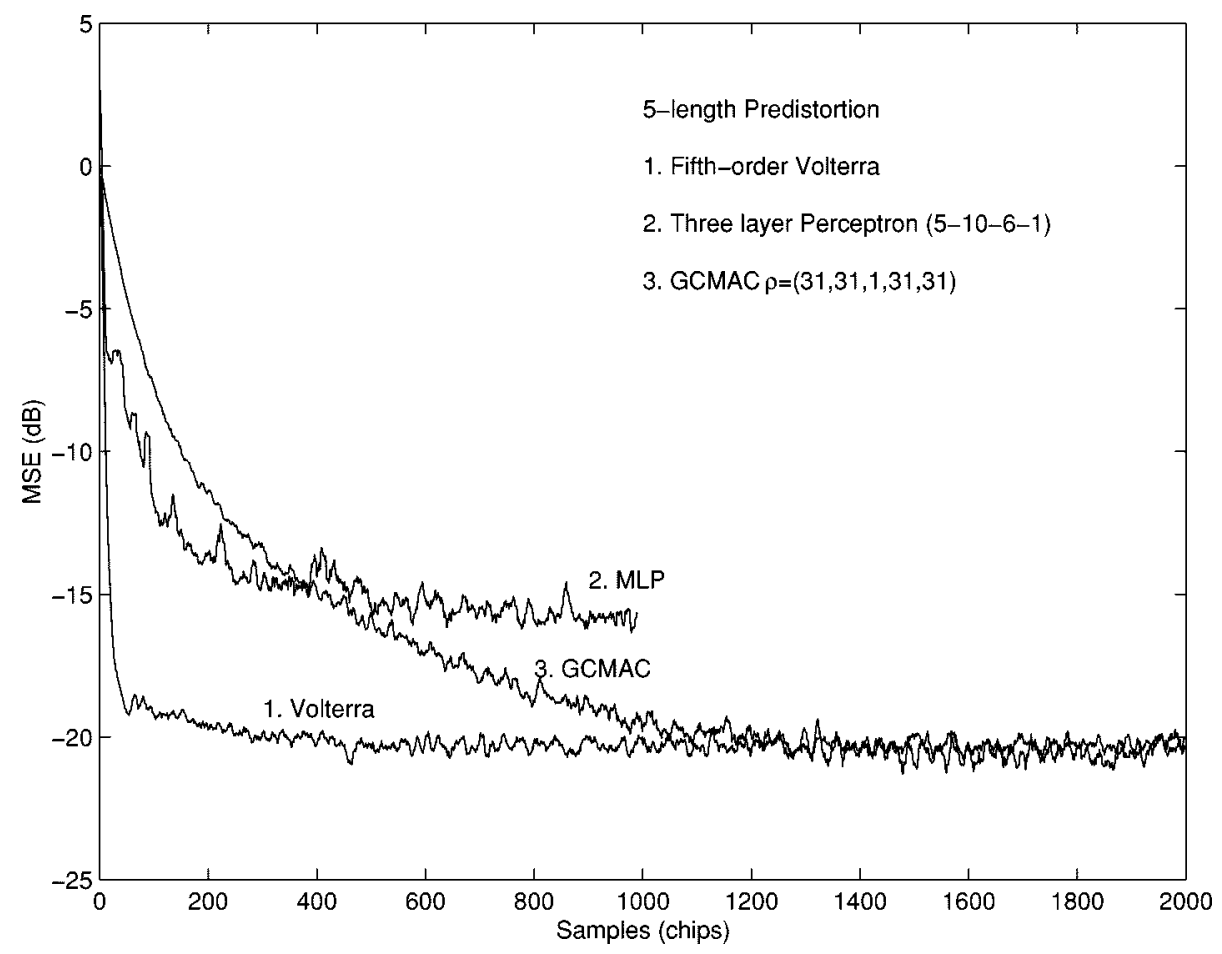

Fig. 6. Convergence of FSDP $(\lambda=8)$ when the amplifier delivers maximum power (saturation).

\section{A. Predistortion Schemes}

The ideal transmitted constellation is given by a linear combination of $N$ QPSK symbols $(N=6)$. The desired user's power is set to unity, and the amplitudes of the remaining users are randomly taken from interval $(0,1)$.

Fig. 6 shows the MSE evolution for different networks acting as fractionally spaced data predistorters $(P=5, \lambda=8)$ when the amplifier is saturated. The compared networks are:

1) $\lambda=8$ : multilayer perceptron (MLP) with five input neurons, ten neurons at the first hidden layer, six neurons at the second hidden layer, and one neuron at the output (5-10-6-1);

2) a fifth-order Volterra filter (VF) $\left(P+P^{3}+P^{5}=3255\right.$ adjustable weights);

3) a GCMAC network with $Q=32, \rho=[31,31,1,31,31]$, and constant LBFs.

It is interesting to observe that the initial convergence speed of both the MLP and the VF is greater than that of the GCMAC. While the MLP and the VF are global approaches (when an individual weight changes, all the output space is modified), the GCMAC performs the approximation locally; therefore, it only produces response on those regions where the initial samples are placed. The main advantage of GCMAC is that the number of floating-point operations (flops) per processed sample $(2 \times$ $\rho_{\max }=62$ flops) is independent of the order $P$. In spite of this, the final MSE remains always below the $-20 \mathrm{~dB}$ level, which produces a negligible impact on the probability of error in a practical CDMA system.

Fig. 7 clarifies the behavior of the GCMAC-based FSDP in the spectrum domain. It represents the power spectrum density of the transmitted CDMA signal when the amplifier delivers maximum output power. Using an FSDP with an oversampling factor $\lambda=8$ (Fig. 7), the sidelobes are reduced in $10 \mathrm{~dB}$ at the adjacent channel frequency $\left(f / f_{\text {chip }}= \pm 1.22\right)$. Higher reductions can be achieved by increasing the oversampling factor, as shown in Table I.

\section{B. Equalization Schemes}

In order to assess the performance of the proposed receivers, we consider an asynchronous CDMA system $\left(\tau_{n, m} \neq 0\right)$. Also, the set of experiments takes into account multiuser interference $(N=6)$ and fading. The level of multiuser interference is specified by the near-far ratio (NFR)

$$
\mathrm{NFR}=\frac{\text { user } n \text { power }}{\text { user } 1 \text { power }}=\frac{A_{n}^{2}}{A_{1}^{2}}
$$

with user 1 being the desired user. As was stated in Section II, two mechanisms of fading are considered: time spreading and time variance of the channel.

For such an scenario, the GCMAC-based DFE (type I) is described by the following parameters: $Q=64$ (6-bit $\mathrm{A} / \mathrm{D})$, $\rho_{i}=63$, and constant LBFs. The order of the GCMAC network is $P=N+N_{b}=7\left(N_{b}=1\right)$, and the length of the linear FIR section is $N_{b}^{\prime}=2$. Initially, the receiver learns the ideal Bayesian solution using a known preamble. After the supervised training, the symbol decision is used as the desired output.

In the first test, we study the convergence and the tracking abilities of the proposed receiver on a time-variant frequency-selective fading channel. We have used the discrete multipath model with exponential power profile specified in ETSI UMTS recommendation 30.06 [3] (see Table II). In the interest of simplicity, delays are taken to be multiples of the chip period $\left(\tau_{n, m} \in\left\{0, T_{c}, 2 T_{c}, \ldots,(L-1) T_{c}\right\}\right)$. 


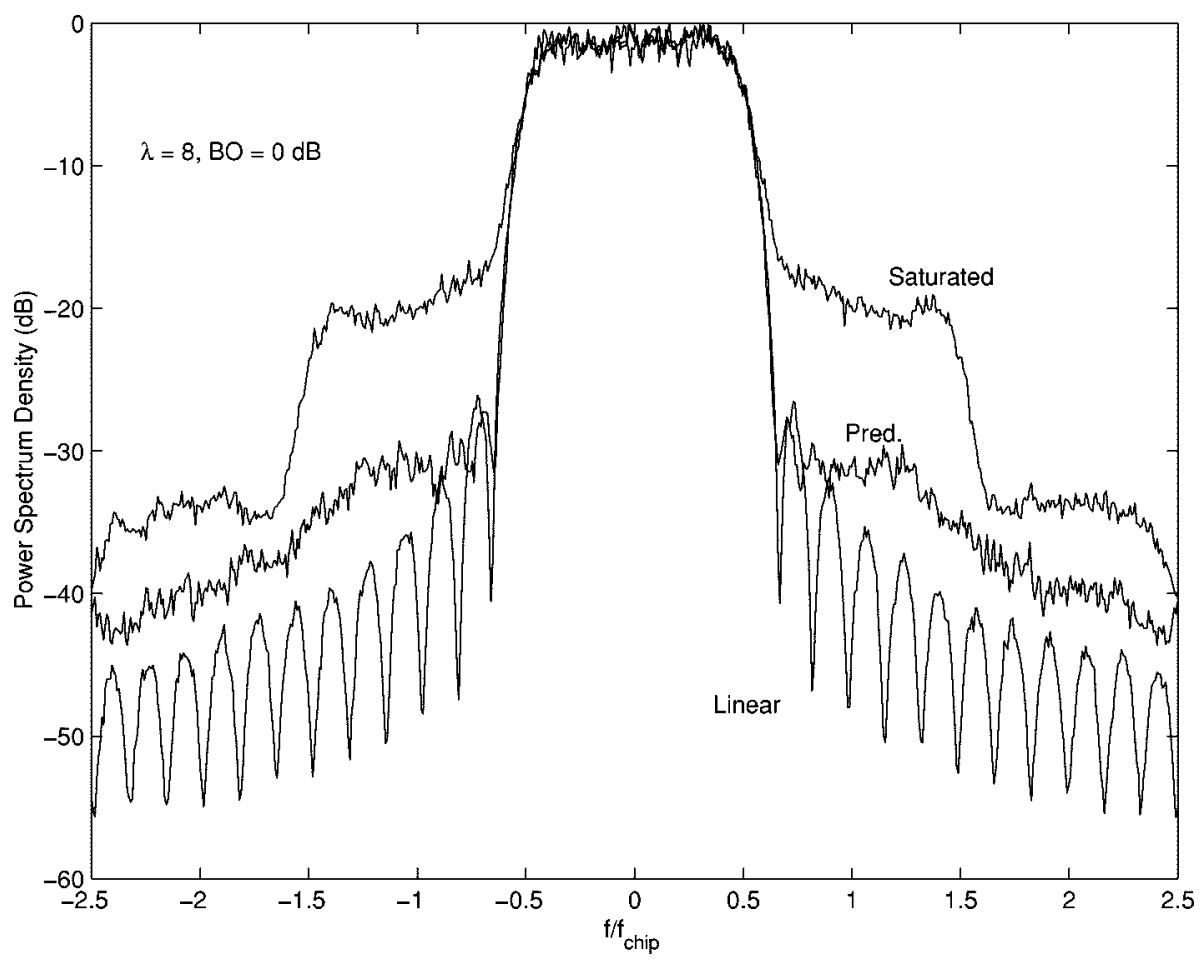

Fig. 7. Transmitted power spectrum density when the amplifier delivers maximum power (saturation). $\lambda=8$.

TABLE I

REDUCTION OF THE SiDE-LOBES LEVEL AT THE FREQUENCY CORRESPONDING TO THE ADJACENT CHANNEL WHEN THE AMPLIFIER IS SATURATED

\begin{tabular}{l||c|c|c|c}
\hline$\lambda$ & 2 & 4 & 8 & 16 \\
\hline Reduction $(\mathrm{dB})$ & 4 & 8 & 10 & 14 \\
\hline
\end{tabular}

TABLE II

EXPOTENTIAL POWER PROFILES SPECIFIED IN ETSI UMTS RECOMMENDATION 30.06

\begin{tabular}{c|c|c}
\hline \multirow{2}{*}{ Taps } & \multicolumn{2}{|c}{ Avg. Power (dB) } \\
\cline { 2 - 3 } & Rician & Rayleigh \\
\hline 1 & 0.0 & -5.0 \\
\hline 2 & -1.0 & 0.0 \\
\hline 3 & -9.0 & -12.8 \\
\hline 4 & -10.0 & -10.0 \\
\hline 5 & -15.0 & -25.2 \\
\hline 6 & -20.0 & -16.0 \\
\hline
\end{tabular}

Fig. 8 depicts the evolution of the bit error rate (BER). The fading channel $\left(E_{b} / N_{0}=10 \mathrm{~dB} ; \mathrm{NFR}_{n}=12 \mathrm{~dB}\right)$ is changed suddenly from Rician (nonfaded line-of-sight signal component) to Rayleigh (direct signal component is faded in 5 dB) after 60 bits. If the training preamble is long enough (30 bit), the GCMAC-based receiver maintains the BER in low values, even after the change in the time-dispersive channel (solid line). However, if the preamble is shortened (15 bits), the result is an initial moderate increase of BER that leads to divergence after the fading.

Another performance measure is the cumulative distribution function (CDF) of BER. In Fig. 9, the vertical axis represents the percentage of multipath fading channels with better BER than the horizontal axis value. Thus, for the DFE Type I equalizer with a feedback order $N_{b}=2$, about $50 \%$ of the multipath channels considered $\left(E_{b} / N_{0}=10 \mathrm{~dB} ; \mathrm{NFR}_{n}=12 \mathrm{~dB}\right)$ have BER $<4 \times 10^{-3}$. This representation gives additional insight into the performance of the proposed receiver scheme on timevariant channels.

\section{CONCLUSION}

Schemes that compensate for distortion in CDMA communications systems at the transmitter (predistorter) and receiver (equalizer) ends have been proposed. Compensators are based on the GCMAC neural network, since it provides accurate approximations with fast convergence and can be easily implemented using general-purpose DSP hardware.

We have used a fractionally spaced data predistorter that modifies the transmitted signal not only at the symbol instants but also at intermediate instants. In this work, the FSDP consists of $\lambda$ GCMAC predistorters in parallel. They provide a new pulse shape that improves the spectral response after the nonlinear processing in the transmission chain. This way, the GCMAC predistorter compensates simultaneously for both the spectral widening and the constellation warping and clustering.

In addition, we have focused on the adaptive interference suppression in a CDMA system. We have proposed two schemes that perform a decision-feedback equalization of multipath channels. The first one processes the whole input vector (which includes both received and previously decided symbols) to produce an estimate of the transmitted symbol. The second scheme is simpler; it uses two different networks that process separately the received samples and the (previously) detected data symbols. Again, the GCMAC network was used to perform the equalization. To improve the convergence of GCMAC, a novel committee machine composed of a linear FIR in parallel with a GCMAC network is proposed. This receiver 


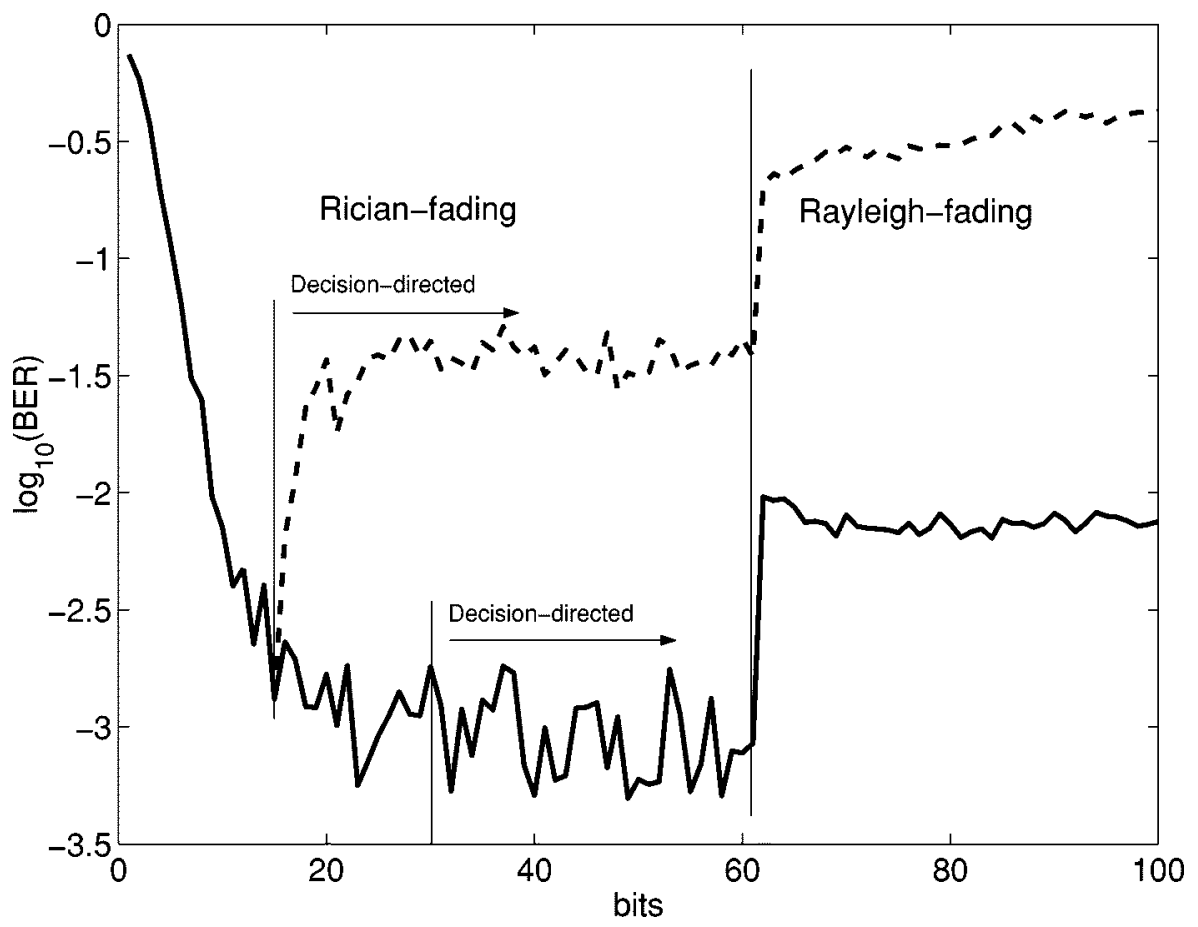

Fig. 8. Evolution of the BER on a time-variant frequency-selective fading channel. Solid line: evolution using a known preamble of 30 bits. Dashed line: preamble of 15 bits. Curves are ensembled averages of 15 independent runs.

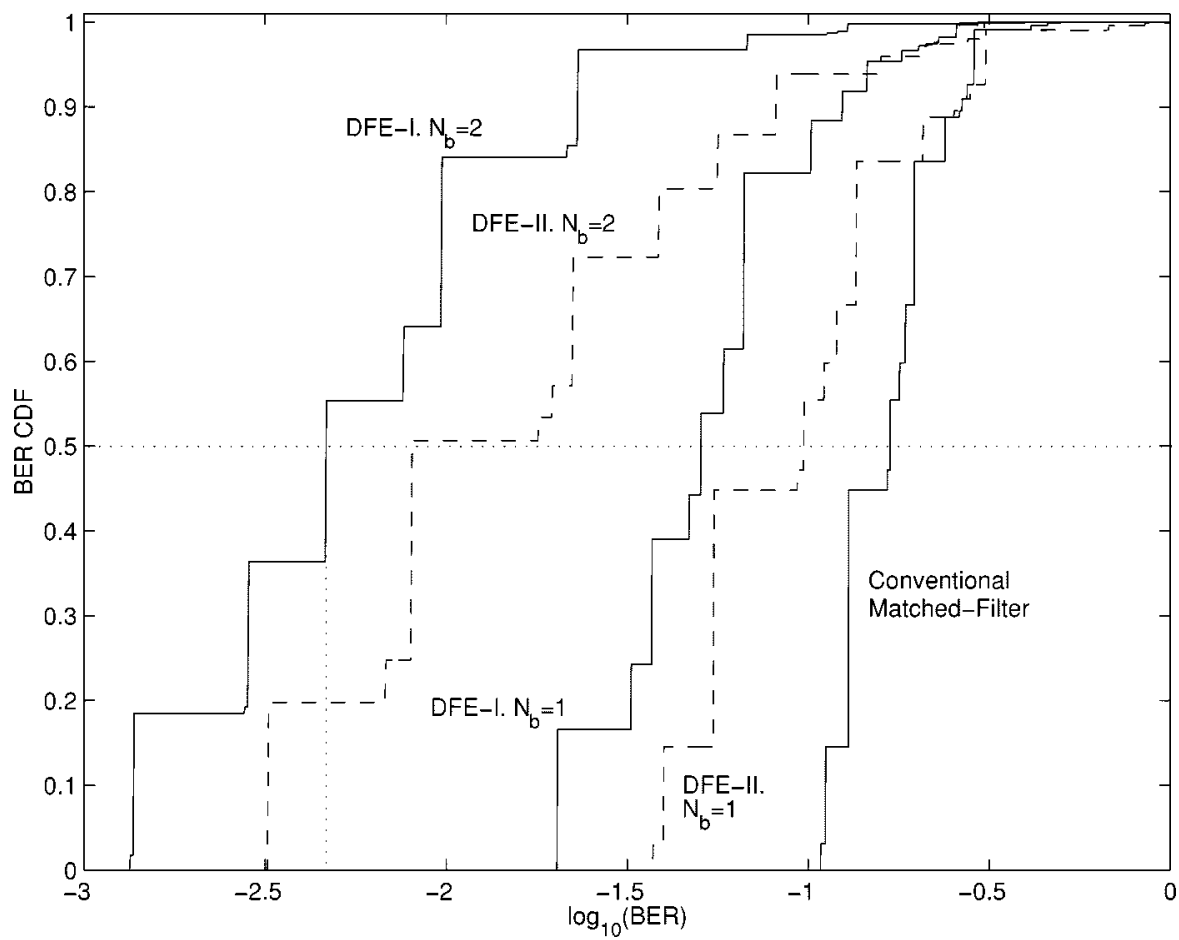

Fig. 9. BER CDF versus values of BER. The vertical axis represents the percentage of multipath fading channels $\left(E_{b} / N_{0}=10 \mathrm{~dB} ; \mathrm{NFR} n=12 \mathrm{~dB}\right)$ with better BER than the horizontal axis value.

obtains an interesting performance in dynamic scenarios where, in addition to multiaccess interference, multipath fading is present.

\section{ACKNOWLEDGMENT}

The authors would like to acknowledge the suggestions of the anonymous reviewers.

\section{REFERENCES}

[1] A. A. M. Saleh and J. Salz, "Adaptive linearization of power amplifiers in digital radio systems," Bell Syst. Tech. J., vol. BSTJ-62, no. 4, pp. 1019-1033, Apr. 1983.

[2] S. Verdú, Multiuser Detection. Cambridge, U.K.: Cambridge Univ. Press, 1998.

[3] 3rd Generation Partnership Project (3GPP), "Technical specification group radio access network; physical layer-General description (3G TSS 25.201. Version 3.00),", Tech. Rep. 3GPP, Dec. 1999. 
[4] A. A. M. Saleh, "Frequency-independent and frequency-dependent nonlinear models of TWT amplifiers," IEEE Trans. Commun., vol. COM-29, pp. 1715-1720, Nov. 1981.

[5] B. Sklar, "Rayleigh fading channels in mobile digital communication systems-Part I: Characterization,” IEEE Commun. Mag., vol. 35, no. 7, pp. 90-100, 1997.

[6] G. Karam and H. Sari, "Data predistortion techniques using intersymbol interpolation," IEEE Trans. Commun., vol. 38, pp. 1716-1723, Oct. 1990.

[7] M. Visintin, "An adaptive (DSP) predistortion for radio systems," Eur. Trans. Telecommun., vol. 5, pp. 95-101, July 1994.

[8] N. Benvenuto, F. Piazza, A. Unicini, and M. Visintin, "Generalized backpropagation algorithm for training a data predistorter with memory in radio systems," Electron. Lett., vol. 32, pp. 1925-1926, Sept. 1996.

[9] K. Das and S. D. Morgera, "Adaptive interference cancellation for (DS-CDMA) systems," IEEE J. Select. Areas Commun., vol. 16, pp. 1774-1784, Dec. 1998

[10] R. T. Causey and J. R. Barry, "Blind multiuser detection using linear prediction," IEEE J. Select. Areas Commun., vol. 16, pp. 1702-1710, Dec. 1998.

[11] J. S. Albus, "A new approach to manipulator control: The cerebellar model articulation controller," J. Dyn. Syst., Meas., Contr., vol. 63, no. 3, pp. 220-227, Sept. 1975

[12] F. J. González-Serrano, A. Artés-Rodríguez, and A. R. Figueiras-Vidal, "Generalizing CMAC architecture and training," IEEE Trans. Neural Networks, vol. 9, Nov. 1998.

[13] J. S. Ker, Y. H. Kuo, R. C. Wen, and B. D. Liu, "Hardware implementation of CMAC neural network with reduced storage requirement," IEEE Trans. Neural Networks, vol. 8, pp. 1545-1556, Nov. 1997.

[14] S. Haykin, Neural Networks: A Comprehensive Foundation, 2nd ed. Englewood Cliffs, NJ: Prentice-Hall, 1998.

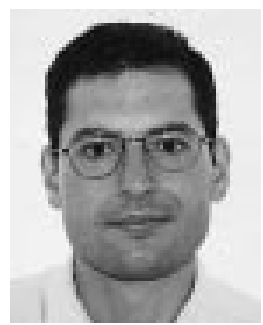

Francisco Javier González-Serrano (M'94) was born in Betanzos, Spain, on October 10, 1968. He received the telecommunication engineering and Ph.D. degrees from the University of Vigo, Spain, in 1992 and 1997, respectively.

From 1992 to 1993 he was with the DSP Research Group, University of Vigo, as a Research Associate. From 1993 to 1998, he was an Assistant and Associate Professor with the University of Vigo. Currently, he is an Associate Professor with the University Carlos III, Madrid, Spain. His research interests are in the areas of digital signal processing, wireless communications, and multimedia communications.

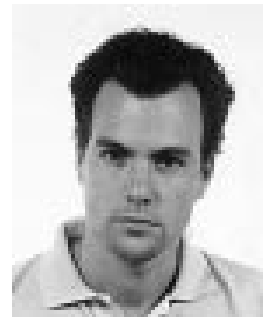

Juan José Murillo-Fuentes ( $\left.\mathrm{S}^{\prime} 00\right)$ received the telecommunication engineering degree from the University of Seville, Spain, in 1996. He is now pursuing the Ph.D. degree from the University Carlos III, Madrid, Spain.

Since 1999, he has been an Assistant Professor at the University Carlos III. His main research interests include neural networks and their applications to communications, array processing with emphasis in algorithms for blind separation of sources and multiuser detection in third-generation systems, and image processing applied to teledetection. 